\title{
Generalized Fibonacci polynomials and Fibonomial coefficients
}

\author{
Tewodros Amdeberhan \\ Department of Mathematics, Tulane University, \\ New Orleans, LA 70118, USA, tamdeber@tulane.edu \\ Xi Chen \\ School of Mathematical Sciences, Dalian University of Technology, \\ Dalian City, Liaoning Province, 116024, P. R. China, xichen.dut@gmail.com* \\ Victor H. Moll \\ Department of Mathematics, Tulane University, \\ New Orleans, LA 70118, USA, vhm@tulane.edu ${ }^{\dagger}$ \\ Bruce E. Sagan \\ Department of Mathematics, Michigan State University, \\ East Lansing, MI 48824, USA, sagan@math.msu.edu
}

July 30, 2013

Key Words: binomial theorem, Catalan number, Dodgson condensation, Euler-Cassini identity, Fibonacci number, Fibonomial coefficient, Lucas number $q$-analogue, valuation

AMS subject classification (2000): Primary 05A10; Secondary 11B39, $11 B 65$.

Running title: Fibonacci polynomials and Fibonomial coefficients

\begin{abstract}
The focus of this paper is the study of generalized Fibonacci polynomials and Fibonomial coefficients. The former are polynomials $\{n\}$ in variables $s, t$ given by $\{0\}=0,\{1\}=1$, and $\{n\}=s\{n-1\}+t\{n-2\}$ for $n \geq 2$. The latter are defined by $\left\{\begin{array}{l}n \\ k\end{array}\right\}=\{n\} ! /(\{k\} !\{n-k\} !)$ where $\{n\} !=\{1\}\{2\} \ldots\{n\}$. These quotients are also polynomials in $s, t$ and specializations give the ordinary binomial coefficients, the Fibonomial coefficients, and the $q$-binomial coefficients. We present some of their fundamental properties, including a more general recursion for $\{n\}$, an analogue of the binomial theorem, a new proof of the Euler-Cassini identity in this setting with applications to estimation of tails of series, and valuations when $s$ and $t$ take on integral values. We also study a corresponding analogue of the Catalan numbers. Conjectures and open problems are scattered throughout the paper.
\end{abstract}

${ }^{*}$ Research partially supported by a grant from the China Scholarship Council

${ }^{\dagger}$ Research partially supported by the National Science Foundation NSF-DMS 1112656 


\section{Introduction}

We will be studying generalized Fibonacci polynomials and generalized Fibonomial coefficients. Throughout this work, $\mathbb{P}$ will stand for the positive integers. The Fibonacci numbers $F_{n}$ are defined by $F_{0}=0, F_{1}=1$ and, for $n \geq 2$,

$$
F_{n}=F_{n-1}+F_{n-2} .
$$

The Lucas numbers $L_{n}$ are defined by the same recurrence, with the initial conditions $L_{0}=2$ and $L_{1}=1$. The reader will find an introduction to these well-studied sequences in the books by Koshy [18] and Moll [23.

One generalization of these numbers which has received much attention is the sequence of Fibonacci polynomials

$$
F_{n}(x)=x F_{n-1}(x)+F_{n-2}(x), \quad n \geq 2,
$$

with initial conditions $F_{0}(x)=0, F_{1}(x)=1$. The generalized Fibonacci polynomials which we will consider depend on two variables $s, t$ and are defined by $\{0\}_{s, t}=0,\{1\}_{s, t}=1$ and, for $n \geq 2$,

$$
\{n\}_{s, t}=s\{n-1\}_{s, t}+t\{n-2\}_{s, t}
$$

Here and with other quantities depending on $s$ and $t$, we will often drop the subscripts if they are clear from context. For example, we have

$$
\{2\}=s, \quad\{3\}=s^{2}+t, \quad\{4\}=s^{3}+2 s t, \quad\{5\}=s^{4}+3 s^{2} t+t^{2} .
$$

When $s$ and $t$ are integers, these sequences were first studied by Lucas in a series of papers [20, 21, 22] and then forgotten. Nearly 100 years later, Hoggatt and Long [16] rediscovered them, this time considering $s$ and $t$ as variables. But they have received considerably less attention than the one variable family in (1), although some of their properties are the same because of the relation

$$
\{n\}_{s, t}=t^{(n-1) / 2} F_{n}\left(\frac{s}{\sqrt{t}}\right) .
$$

Part of the purpose of the present work is to rectify this neglect.

Our notation is chosen to reflect two important specializations of this sequence (other than the one $s=t=1$ already mentioned). In particular, if $s=2$ and $t=-1$ then $\{n\}=n$. And if $s=q+1$ and $t=-q$ then

$$
\{n\}=1+q+q^{2}+\cdots+q^{n-1}=[n]_{q},
$$

the standard $q$-analogue of $n$.

There is a corresponding extension of the Lucas numbers, the generalized Lucas polynomials, defined by

$$
\langle n\rangle_{s, t}=s\langle n-1\rangle_{s, t}+t\langle n-2\rangle_{s, t}, \quad n \geq 2
$$

together with the initial conditions $\langle 0\rangle_{s, t}=2$ and $\langle 1\rangle_{s, t}=s$. Here is a list of the first few polynomials

$$
\langle 2\rangle_{s, t}=s^{2}+2 t, \quad\langle 3\rangle_{s, t}=s^{3}+3 s t, \quad\langle 4\rangle_{s, t}=s^{4}+4 s^{2} t+2 t^{2}, \quad\langle 5\rangle_{s, t}=s^{5}+5 s^{3} t+5 s t^{2} .
$$

Of course, when $s=t=1$ these reduce to the ordinary Lucas numbers.

One can find algebraic expressions for these polynomials using standard techniques from the theory of recursively defined sequences. In particular, the characteristic polynomial of the recurrence is $z^{2}-s z-t$ whose roots are

$$
X=\frac{s+\sqrt{s^{2}+4 t}}{2} \text { and } Y=\frac{s-\sqrt{s^{2}+4 t}}{2} .
$$




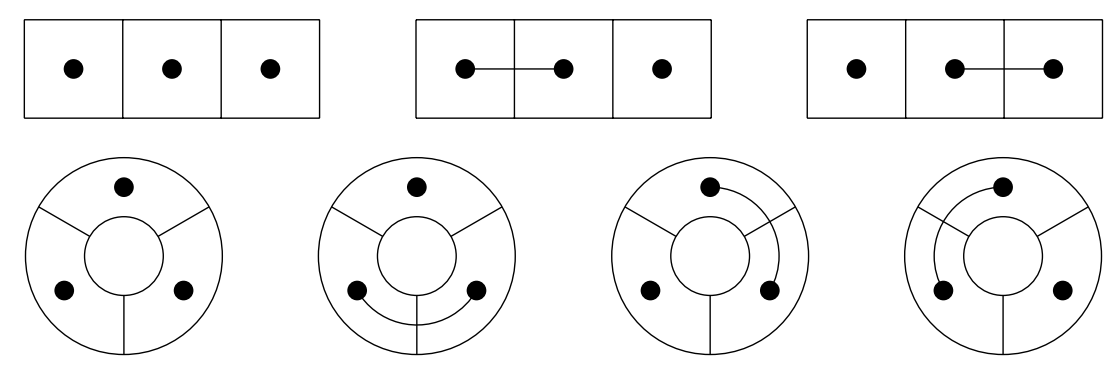

Figure 1: Linear and circular tilings

We will often abbreviate $\sqrt{s^{2}+4 t}=\Delta_{s, t}$. The following relations between $s, t$ and $X, Y$ will be useful

$$
s=X+Y, \quad t=-X Y, \quad \Delta=X-Y .
$$

Any solution of (2) can be expressed as $c X^{n}+d Y^{n}$ for constants $c, d$ depending on the initial conditions. Computing the constants in the two cases of interest to us gives the following analogue of Binet's formula for the Fibonacci numbers.

Proposition 1.1. For $n \geq 0$ we have

$$
\{n\}=\frac{X^{n}-Y^{n}}{X-Y} \quad \text { and } \quad\langle n\rangle=X^{n}+Y^{n} .
$$

Combining this result with equation (3), we see that there is another relation between $\{n\}$ and $[n]$, namely

$$
\{n\}_{s, t}=Y^{n-1}[n]_{X / Y}
$$

This will be useful in the sequel.

In addition to this algebraic approach to our polynomials, there is a combinatorial interpretation derived from the standard interpretation of $F_{n}$ via tiling. A linear tiling, $T$, of a row of squares is a covering of the squares with dominos (which cover two squares) and monominos (which cover one square). We let

$$
\mathcal{L}_{n}=\{T: T \text { a linear tiling of a row of } n \text { squares }\} .
$$

The three tilings in the first row of Figure 1 are the elements of $\mathcal{L}_{3}$. We will also consider circular tilings where the (deformed) squares are arranged in a circle. We will use the notation $\mathcal{C}_{n}$ for the set of circular tilings of $n$ squares. So the set of tilings in the bottom row of Figure 1 is $\mathcal{C}_{3}$. For any type of nonempty tiling, $T$, we define its weight to be

$$
\text { wt } T=s^{\# \text { of monominos in } T} t^{\# \text { of dominos in } T} .
$$

We give the empty tiling $\epsilon$ of zero boxes the weight wt $\epsilon=1$ if it is being considered as a linear tiling or wt $\epsilon=2$ if it is being considered as a circular tiling. The following proposition is immediate from the definitions of weight and of our generalized polynomials.

Proposition 1.2. For $n \geq 0$ we have

$$
\{n+1\}=\sum_{T \in \mathcal{L}_{n}} \mathrm{wt} T \quad \text { and }\langle n\rangle=\sum_{T \in \mathcal{C}_{n}} \mathrm{wt} T .
$$

We are now in a position to define corresponding generalized binomial coefficients. Given a sequence $\mathbf{a}=\left\{a_{n}\right\}$ of nonzero real numbers, it is natural to define the a-factorials by

$$
n !_{\mathbf{a}}=\prod_{i=1}^{n} a_{i}
$$


and the a-binomial coefficients by

$$
\left(\begin{array}{l}
n \\
k
\end{array}\right)_{\mathbf{a}}=\frac{n !_{\mathbf{a}}}{k !_{\mathbf{a}}(n-k) !_{\mathbf{a}}}
$$

The classical example comes from $a_{n}=n$ with the standard $q$-analogue being obtained when $a_{n}=[n]_{q}$. Here, we will consider $a_{n}=\{n\}_{s, t}$ and $a_{n}=\langle n\rangle_{s, t}$. To simplify the notation, these are written, respectively, as

for factorials, and

$$
\{n\}_{s, t} ! \quad \text { and }\langle n\rangle_{s, t} \text { ! }
$$

$$
\left\{\begin{array}{l}
n \\
k
\end{array}\right\}_{s, t} \quad \text { and } \quad\left\langle\begin{array}{l}
n \\
k
\end{array}\right\rangle_{s, t}
$$

for binomial coefficients. The product $\{n\}$ ! is called a generalized fibotorial and $\langle n\rangle$ ! is a generalized lucatorial. The binomial coefficients $\left\{\begin{array}{l}n \\ k\end{array}\right\}$ and $\left\langle\begin{array}{l}n \\ k\end{array}\right\rangle$ are called generalized Fibonomial and Lucanomial coefficients, respectively.

We can relate the generalized Fibonomials to the $q$-binomial coefficients algebraically. Indeed, it follows easily from (6) that

$$
\left\{\begin{array}{l}
n \\
k
\end{array}\right\}_{s, t}=Y^{k(n-k)}\left[\begin{array}{l}
n \\
k
\end{array}\right]_{X / Y} .
$$

There is also a simple combinatorial interpretation of $\left\{\begin{array}{l}n \\ k\end{array}\right\}$ which was given by Savage and Sagan [24] using tilings of a $k \times(n-k)$ rectangle containing a partition. But we will not use this description, and instead we refer the reader to their paper for the details. It would be very interesting to give combinatorial proofs for some of the results we give for Fibonomials.

The rest of this paper is structured as follows. In the next section we present some of the fundamental properties of $\{n\}$ and $\langle n\rangle$ which will be useful in the rest of the paper. Section 3 is devoted to valuations. In particular, we provide a complete description of the 2-adic valuation of $\{n\}$ and $\{n\}$ ! for arbitrary integers $s, t$. The Euler-Cassini identity is the focus of Section 4 . We give a new proof of the diagonal case of this equality using Dodgson condensation. We also use this identity to give estimates for the tails of Fibonacci analogues of the series for the Riemann zeta function. In Section 5 we return to the generalized Fibonomial coefficients and consider their recursions and analogues of the binomial theorem. We end by studying an $s, t$-version of the Catalan numbers $C_{n}$ and proving analogues of the theorem giving the 2-adic valuation of $C_{n}$. Various open problems and conjectures are mentioned.

\section{Fundamental properties of $\{n\}$ and $\langle n\rangle$}

In this section we collect some of the important properties of $\{n\}_{s, t}$ and $\langle n\rangle_{s, t}$ to be used in the sequel. Most can be proved using either the algebraic descriptions in terms of $X$ and $Y$, or the combinatorial interpretations, or both. Often the demonstrations do not differ significantly from ones already in the literature, so we will sometimes omit the proofs and just supply references where they can be found.

We begin with the expansion of our polynomials into monomials.

Proposition 2.1. The polynomials $\{n\}$ and $\langle n\rangle$ are given by

$$
\{n\}=\sum_{k \geq 0}\left(\begin{array}{c}
n-k-1 \\
k
\end{array}\right) s^{n-2 k-1} t^{k}
$$

and

$$
\langle n\rangle=\sum_{k \geq 0} \frac{n}{n-k}\left(\begin{array}{c}
n-k \\
k
\end{array}\right) s^{n-2 k} t^{k} .
$$


Proof. We just prove the first identity as the second is similar. By Proposition 1.2, it suffices to show that the $k$ th term of the sum is the sum of the weights of all linear tilings of $n-1$ squares with $k$ dominos. If there are $k$ dominos, then there must be $n-2 k-1$ monominos and so this accounts for the monomial $s^{n-2 k-1} t^{k}$. To count the number of arrangements of these tiles, number the squares $1, \ldots, n-1$ from left to right. Then picking the places for the left endpoints of the dominos is equivalent to picking $k$ numbers from $1, \ldots, n-2$ with no two consecutive. The number of ways of doing this is $\left(\begin{array}{c}n-k-1 \\ k\end{array}\right)$, and once the dominos are placed, there is no further choice for distributing the monominos. This finishes the proof.

The next result is a useful generalization of the defining recurrence for the polynomials $\{n\}$.

Theorem 2.2. For $m \geq 1$ and $n \geq 0$ we have

$$
\{m+n\}=\{m\}\{n+1\}+t\{m-1\}\{n\} .
$$

Proof. This can be given a combinatorial proof (see [24]), but we will indicate an algebraic one to illustrate the method. First one uses (5) and Proposition 1.1 to convert the equality into an equivalent statement about polynomials in $X$ and $Y$. This statement can then be easily verified by algebraic manipulations.

We now note two identities relating $\{n\}$ and $\langle n\rangle$.

Proposition $2.3([24])$. For $n \geq 1$ we have

$$
\langle n\rangle=\{n+1\}+t\{n-1\}
$$

And for $m, n \geq 0$ we have

$$
\{m+n\}=\frac{\langle m\rangle\{n\}+\{m\}\langle n\rangle}{2} .
$$

The next result can be used to show that many divisibility properties carry over directly from the integers $n$ to the polynomials $\{n\}$.

Proposition 2.4 ([16]). For $m, n \geq 1$ we have

$$
\operatorname{gcd}(\{m\},\{n\})=\{\operatorname{gcd}(m, n)\} .
$$

Equivalently, $m$ divides $n$ if and only if $\{m\}$ divides $\{n\}$.

Using standard techniques, one can convert the defining recursion for $\{n\}$ into a generating function.

Proposition 2.5. The generating function of the polynomials $\{n\}$ is given by

$$
\sum_{n=0}^{\infty}\{n\} z^{n}=\frac{z}{1-s z-t z^{2}} .
$$

As an application of this result, we will derive a generalization of the following well-known identity for Fibonacci numbers

$$
\sum_{n=0}^{\infty} \frac{F_{n}}{2^{n+1}}=1
$$

which is the special case $s=t=1$ and $z=1 / 2$ of the above proposition. 
Corollary 2.6. For $s, t \in \mathbb{P}$ we have

$$
\sum_{n=0}^{\infty} \frac{t\{n\}_{s, t}}{(s+t)^{n+1}}=\frac{1}{s+t-1}
$$

Proof. We will give both algebraic and combinatorial proofs. The former is obtained by setting $z=$ $1 /(s+t)$ in the generating function of Proposition 2.5. We must make sure that this substitution is analytically valid in that $1 /(s+t)$ is smaller than the radius of convergence of the power series which is $\min \{1 /|X|, 1 /|Y|\}$. But this is a routine check using equation (41). Once the substitution is made, simple algebraic manipulations complete the demonstration.

For the combinatorial proof, consider an infinite row of squares numbered left to right by the positive integers. Suppose each square can be colored with one of $s$ shades of white and $t$ shades of black. Let $Z$ be the random variable which returns the box number at the end of the first odd-length block of boxes all of the same black shade. For $n \in \mathbb{P}$, the event $Z=n$ is equivalent to having box $n$ painted with one of the shades of black, box $n+1$ painted with any of the remaining colors, and all blocks of a black shade among the first $n-1$ squares being of even length. So there are $t$ choices for the color of box $n$ and $s+t-1$ choices for the color of box $n+1$. Each coloring of the first $n-1$ squares gives rise to a tiling where each white box is replaced by a monomino and a block of $2 k$ boxes of the same black shade is replaced by $k$ dominoes. Also, the weight of the tiling is just the number of colorings mapping to it. Thus, by Proposition 1.2, the number of colorings for the first $n-1$ boxes is $\{n\}$. Hence

$$
P(Z=n)=\frac{t(s+t-1)\{n\}_{s, t}}{(s+t)^{n+1}} .
$$

Summing these probabilities finishes the proof.

We end this section by exploring the binomial transformation of the sequence $\{n\}, n \geq 0$. Interestingly, doing so involves a change of variables from $s, t$ to $s+2, t-s-1$. We then use the transform to prove a well-known identity for Fibonacci numbers.

Proposition 2.7. For $n \geq 0$ we have

$$
\sum_{k=0}^{n}\left(\begin{array}{l}
n \\
k
\end{array}\right)\{k\}_{s, t}=\{n\}_{s+2, t-s-1}
$$

In particular, for $s=t=1$,

$$
\sum_{k=0}^{n}\left(\begin{array}{l}
n \\
k
\end{array}\right) F_{k}=F_{2 n}
$$

Proof. Using Proposition 1.1, we have the exponential generating function

$$
\sum_{k=0}^{\infty}\{k\}_{s, t} \frac{z^{k}}{k !}=\frac{e^{X z}-e^{Y z}}{X-Y}
$$

Note that

$$
X+1, Y+1=\frac{(s+2) \pm \sqrt{s^{2}+4 t}}{2}=\frac{(s+2) \pm \sqrt{(s+2)^{2}+4(t-s-1)}}{2} .
$$

Putting everything together and using the product rule for exponential generating functions gives

$$
\sum_{n=0}^{\infty} \frac{z^{n}}{n !} \sum_{k=0}^{n}\left(\begin{array}{l}
n \\
k
\end{array}\right)\{k\}_{s, t}=e^{z} \frac{e^{X z}-e^{Y z}}{X-Y}=\frac{e^{(X+1) z}-e^{(Y+1) z}}{X-Y}=\sum_{n \geq 0}\{n\}_{s+2, t-s-1} \frac{z^{n}}{n !} .
$$


Extracting the coefficients of $z^{n} / n$ ! completes the proof of the first equation.

For the second, from what we have just proved it suffices to show that $\{n\}_{3,-1}=\{2 n\}_{1,1}$. But

$$
\{2 n\}_{1,1}=\frac{1}{\sqrt{5}}\left[\left(\frac{1+\sqrt{5}}{2}\right)^{2 n}-\left(\frac{1-\sqrt{5}}{2}\right)^{2 n}\right]=\frac{1}{\sqrt{5}}\left[\left(\frac{3+\sqrt{5}}{2}\right)^{n}-\left(\frac{3-\sqrt{5}}{2}\right)^{n}\right]=\{n\}_{3,-1}
$$

and we are done.

\section{$3 \quad$ Arithmetic properties}

We will be concerned with the $d$-adic valuation function

$$
\nu_{d}(n)= \begin{cases}\text { the highest power of } d \text { dividing } n & \text { if } n \neq 0 \\ \infty & \text { if } n=0\end{cases}
$$

If the subscript is missing, then it is assumed that $d=2$. A fact about valuations which we will use repeatedly is that if $\nu_{d}(m) \neq \nu_{d}(n)$ then

$$
\nu_{d}(m+n)=\min \left\{\nu_{d}(m), \nu_{d}(n)\right\}
$$

Our primary goal will be to characterize $\nu_{2}\left(\{n\}_{s . t}\right)$ for all possible integers $s, t$. This will then be used in Section [6 to give analogues of a well-known theorem about the 2-adic valuation of the Catalan numbers. We will end the section with an indication of what can be said for other moduli.

We will now characterize $\nu(\{n\})=\nu_{2}\left(\{n\}_{s, t}\right)$ for all integral $s, t$ as well as $\nu(\{n\} !)$. We first consider the case when both $s, t$ are odd. If $S$ is a set of integers then we will have much use for the indicator function

$$
\delta_{S}(k)= \begin{cases}1 & \text { if } k \in S \\ 0 & \text { if } k \notin S\end{cases}
$$

In this context, we will let $E$ and $O$ stand for the even and odd integers, respectively.

Lemma 3.1. Let $s$ and $t$ be odd. We have $\nu(\{n\})=0$ whenever $n=3 k+1$ or $3 k+2$. If $n=3 k$ then

$$
\nu(\{3 k\})=\left\{\begin{array}{lll}
1+\delta_{E}(k)(\nu(k\{6\})-2) & \text { if } t \equiv 1 & (\bmod 4), \\
\nu(k\{3\}) & \text { if } t \equiv 3 & (\bmod 4) .
\end{array}\right.
$$

Proof. Our proof will be by induction on $n$ where the base cases are easy to verify. From the recursion

$$
\{n\}=s\{n-1\}+t\{n-2\}
$$

and fact that $s$ and $t$ are odd, it is clear that both $\{3 k+1\}$ and $\{3 k+2\}$ are odd while $\{3 k\}$ is even. This finishes the cases when $n=3 k+1$ or $n=3 k+2$. The demonstrations when $n$ is divisible by 3 are similar for both possible residues of $t$, so we will only present $t \equiv 3(\bmod 4)$.

Suppose now that $n=6 k+3$ for some integer $k$. Using the recursion in Theorem 2.2 we have

$$
\{6 k+3\}=\{3\}\{6 k+1\}+t\{2\}\{6 k\} .
$$

By hypothesis and induction we know that $\{6 k+1\},\{2\}$, and $t$ are odd. Furthermore, by induction again, $\nu(\{6 k\})=1+\nu(k\{3\})>\nu(\{3\})$. So using (9) on the previous displayed equation gives $\nu(\{6 k+3\})=\nu(\{3\})=\nu((n / 3)\{3\})$ since $n / 3$ is odd. This is the desired conclusion. 
For the final case, let $n=6 k+6$ for some $k$. Using Theorem 2.2 repeatedly we obtain $\{6 k+6\}=\{3 k+4\}\{3 k+3\}+t\{3 k+2\}\{3 k+3\}=\{3 k+3\}\left(\left(s^{3}+3 s t\right)\{3 k+1\}+\left(s^{2} t+2 t^{2}\right)\{3 k\}\right)$.

As before, we can ignore $\{3 k+1\}$ and factors of $s$ or $t$ since they are odd. Since $s$ is odd, $s^{2} \equiv 1$ $(\bmod 4)$. It follows that $\nu\left(s^{2}+3 t\right)=1$ while $\nu(\{3\})=\nu\left(s^{2}+t\right) \geq 2$. Applying (9) and induction to the previous displayed equation gives

$$
\nu(\{6 k+6\})=\nu(\{3 k+3\})+1=\nu((k+1)\{3\})+1=\nu((2 k+2)\{3\})=\nu((n / 3)\{3\})
$$

which is, again, what we want.

We can use this lemma to calculate the 2-adic valuation of the corresponding factorials.

Corollary 3.2. Let $s$ and $t$ be odd. We have

$$
\nu(\{n\} !)=\left\{\begin{array}{lll}
\left.\nu(\lfloor n / 3\rfloor !)+\lfloor n / 6\rfloor \nu(\{6\})+\delta_{O}(\lfloor n / 3)\rfloor\right) & \text { if } t \equiv 1 & (\bmod 4), \\
\nu(\lfloor n / 3\rfloor !)+\lfloor n / 3\rfloor \nu(\{3\}) & \text { if } t \equiv 3 & (\bmod 4),
\end{array}\right.
$$

where $\lfloor\cdot\rfloor$ is the floor function.

Proof. Again, we will only provide a proof when $t \equiv 3(\bmod 4)$ as the other congruence class is similar. Write $n=3 k+r$ where $0 \leq r<3$. Using Lemma 3.1 we have

$$
\nu(\{n\} !)=\sum_{i=1}^{n} \nu(\{i\})=\sum_{i=1}^{k} \nu(\{3 i\})=\sum_{i=1}^{k} \nu(i\{3\})=\nu(k !)+k \nu(\{3\})
$$

and using the fact that $k=\lfloor n / 3\rfloor$ finishes the demonstration.

Now we turn to the cases when $s$ and $t$ are of opposite parity. If $s$ is odd and $t$ is even then a simple induction shows that $\{n\}$ is always odd for $n \geq 1$. The reverse case is more interesting.

Lemma 3.3. Let $s$ be even and $t$ be odd. We have

$$
\nu(\{n\})= \begin{cases}\nu(\text { sn/2) } & \text { if } n \text { is even, } \\ 0 & \text { if } n \text { is odd. }\end{cases}
$$

Proof. This proof is much like the one for Lemma 3.1 and so we will content ourselves with stating the main equation for the induction step on even integers

$$
\{2 n\}=\{n\}(s\{n\}+2 t\{n-1\}) .
$$

The reader can easily fill in the details.

The proof of the following corollary is much like that of Corollary 3.2 and so is omitted.

Corollary 3.4. Let $s$ be even and $t$ be odd. We have

$$
\nu(\{n\} !)=\nu(n !)+\lfloor n / 2\rfloor \nu(s / 2) .
$$

Finally, we have the case where both parameters are even. To describe the 2-adic valuations we will rely on a recursion. 
Lemma 3.5. Let $s, t$ be odd. We have

$$
\nu\left(\{n\}_{2^{a} s, 2 t}\right)= \begin{cases}\lfloor n / 2\rfloor+\delta_{4 \mathbb{Z}}(n)\left[\nu\left(n\{4\}_{2 s, 2 t} / 4\right)-2\right] & \text { if } a=1, \\ \lfloor n / 2\rfloor+\delta_{E}(n)[\nu(n)+a-2] & \text { if } a \geq 2 .\end{cases}
$$

Now suppose $s, t$ are arbitrary integers. We have

$$
\nu\left(\{n\}_{2 s, 4 t}\right)=n-1+\nu\left(\{n\}_{s, t}\right) .
$$

This completely determines the 2-adic valuations of $\{n\}$ where both subscripts are even.

Proof. To prove (12) the usual ideas come into play. The equations which are used for the induction are the defining recursion, equation (11), and

$$
\{8 k+4\}_{s, t}=\{4\}_{s, t}\{8 k+1\}_{s, t}+t\{3\}_{s, t}\{8 k\}_{s, t} .
$$

The proof of equation (13) is very simple. In fact, a straightforward induction on $n$ shows that $\{n\}_{2 s, 4 t}=2^{n-1}\{n\}_{s, t}$ which implies the desired result.

For the last statement, by repeated use of equation (13), one can reduce finding $\nu(\{n\})$ to finding $\nu\left(\{n\}_{s . t}\right)$ where either at least one of $s, t$ is odd or both are even and $t$ is twice an odd number. In the former case, the computation is finished by one of our former results. In the latter case, one can use equation (12) to complete the evaluation.

Because of the recursive nature of these valuations, the corresponding formulas for $\nu(\{n\}$ !) are complicated and too messy to be of real interest. On the other hand, we do not wish to give the impression that one can only say interesting things for the modulus $d=2$. So our last result in this section will be for arbitrary $d$.

Proposition 3.6. Consider any positive integer $d \geq 2$. We have, for any $n \geq 1$,

$$
\nu_{d}\left(\{n\}_{d,-1}\right)=\delta_{E}(n) \nu_{d}(d n / 2) .
$$

Proof. First, consider the case where $d$ is a prime. Using the defining recursion for $\{n\}=\{n\}_{d,-1}$ one easily sees that $\{n\}$ is divisible by $d$ if and only if $n$ is even. This completes the $n$ odd case. For even integers, letting $s=d$ and $t=-1$ in equation (8) and re-indexing gives

$$
(-1)^{n-1}\{2 n\}=\sum_{k \geq 0}\left(\begin{array}{c}
n+k \\
2 k+1
\end{array}\right) d^{2 k+1}(-1)^{k}=d n+d n \sum_{k \geq 1} \frac{c_{k} d^{2 k}}{(2 k+1) !}
$$

where the $c_{k}$ are integers because $n$ divides $(n+k)(n+k-1) \ldots(n-k)$. So, by equation (9) , it suffices to show that the $d$-adic valuation of every term in the sum over positive $k$ is at least 1 . Since $d$ is prime, we can use Legendre's well-known formula $\nu_{d}(n !)=\sum_{i \geq 1}\left\lfloor n / d^{i}\right\rfloor$ to show that

$$
\nu_{d}((2 k+1) !) \leq \begin{cases}\frac{2 k}{d-1} & \text { if } d \geq 3 \\ 2 k-1 & \text { if } d=2\end{cases}
$$

From this, it is easy to verify that $\nu_{d}\left(d^{2 k} /(2 k+1) !\right) \geq 1$ which completes the case when $d$ is prime.

To finish the proof, note first that the only place where we used the fact that $d$ was prime was in deriving the upper bounds on $\nu_{d}((2 k+1) !)$. But these will still hold when $d$ is a prime power, and may even become sharper. Finally, for general $d$ we just use the fact that if $p$ and $q$ are relatively prime then $\nu_{p q}(n)=\min \left\{\nu_{p}(n), \nu_{q}(n)\right\}$ for any integer $n$. 
We conjecture that the roles of the modulus $d$ and the parameter $s$ in the previous proposition can be decoupled.

Conjecture 3.7. Suppose $s \geq 2$ is an integer and $d \geq 3$ is an odd integer. There exist positive integers $s^{*}, d^{*}$ depending only on $s, d$ such that $d^{*} \leq d$ and

$$
\nu_{d}\left(\{n\}_{s,-1}\right)=\delta_{d^{*} \mathbb{Z}}(n) \nu_{d}\left(s^{*} n / d^{*}\right) .
$$

Of course, it would be desirable to have a way of computing $s^{*}$ and $d^{*}$ from $s$ and $d$ rather than just an existential proof. The following table lists pairs $\left(s^{*}, d^{*}\right)$ for small values of $s$ and $d$ indexing the rows and columns, respectively. Note that any two values of $s^{*}$ with the same valuation modulo $d$ will yield the same result on the right-hand side of the equation in the conjecture. Also note that if the first positive integer $n$ with $\nu_{d}\left(\{n\}_{s,-1}\right) \neq 0$ is $n=d$ then we have a choice as to whether to let $d^{*}=1$ or $d^{*}=d$.

\begin{tabular}{l|cccc}
$s \backslash d$ & 3 & 5 & 7 & 9 \\
\hline 2 & $(1,1)$ & $(1,1)$ & $(1,1)$ & $(1,1)$ \\
3 & $(3,2)$ & $(1,1)$ & $(7,4)$ & $(9,6)$ \\
4 & $(1,1)$ & $(5,3)$ & $(7,4)$ & $(1,1)$ \\
5 & $(1,1)$ & $(5,2)$ & $(1,1)$ & $(1,1)$
\end{tabular}

\section{The Euler-Cassini identity}

In this section we will consider various results related to the famous Euler-Cassini identity for Fibonacci numbers. We will first recall a version of this equation proved by Cigler [7] for a $q$ analogue of our generalized Fibonacci polynomials. We then show how Dodgson condensation [12] can be used to prove a particular case of this identity. Finally, we use a slightly more general form of Euler-Cassini to give estimates for the tails of certain infinite series with terms involving the polynomials $\{n\}$ evaluated at various integers.

The $q$-analogue of $\{n\}$ which we will be considering is $\{n\}(q)=\{n\}_{s, t}(q)$ defined by $\{0\}(q)=0$, $\{1\}(q)=1$ and

$$
\{n\}(q)=s\{n-1\}(q)+t q^{n-2}\{n-2\}(q),
$$

for $n \geq 2$. Cigler [6, 7, 8, 9] introduced and studied these polynomials which have also been considered by Goyt and Sagan [15] and Goyt and Mathisen [14].

To motivate the (generalized) Euler-Cassini identity, recall that a sequence of real numbers $\left(a_{n}\right)_{n \geq 0}$ is called log concave if it satisfies

$$
a_{n}^{2}-a_{n-1} a_{n+1} \geq 0
$$

for all $n \geq 1$. Many sequences of combinatorial nature are log concave, for example any row of Pascal's triangle will do. The identity

$$
F_{r n}^{2}-F_{r(n+1)} F_{r(n-1)}=(-1)^{r} F_{r}^{2}
$$

shows that the sequence with $a_{n}=F_{r n}$ is $\log$ concave for $r$ even. See the articles of Brenti [4], Stanley [26], or Wilf [27] for more details about log concavity and related issues.

It is easy to see that, for sequences $\left(a_{n}\right)_{n \geq 0}$ of positive reals, the log-concavity condition is equivalent to the seemingly stronger statement that

$$
a_{n} a_{n+m-1} \geq a_{n-1} a_{n+m}
$$

for all $m, n \geq 1$. The importance of the following generalization of the Euler-Cassini identity (which is the special case $s=t=q=1$ ) should now be clear. 
Theorem $4.1([7])$. We have

$$
\{n\}_{s, t}(q) \cdot\{n+m-1\}_{s, q t}(q)-\{n-1\}_{s, q t}(q) \cdot\{n+m\}_{s, t}(q)=(-t)^{n-1} q^{\left(\begin{array}{c}
n \\
2
\end{array}\right)}\{m\}_{s, q^{n} t}(q) .
$$

Note that as an immediate corollary, the sequence $\{n\}_{s, t}$ is $\log$ concave for all $t \leq 0$.

We are going to give a novel proof for the $m=1$ case of this theorem using the Dodgson condensation technique for computing determinants. So we will need a determinantal expression for $\{n\}(q)$. We obtain this using a tri-diagonal matrix which is a method common in the theory of special functions.

Proposition 4.2. The polynomial $\{n\}(q)$ is given by

$$
\{n\}(q)=\operatorname{det}\left(\begin{array}{cccccc}
s & -1 & 0 & \cdots & 0 & 0 \\
q t & s & -1 & \cdots & 0 & 0 \\
0 & q^{2} t & s & \cdots & 0 & 0 \\
\vdots & \vdots & \vdots & \ddots & \vdots & \vdots \\
0 & 0 & 0 & \cdots & s & -1 \\
0 & 0 & 0 & \cdots & q^{n-2} t & s
\end{array}\right)
$$

where the tridiagonal matrix is of size $(n-1) \times(n-1)$.

Proof. By expansion about the last column, one easily verifies that the determinant satisfies the same initial conditions and recurrence as $\{n\}(q)$.

For the final piece of background, we recall the method of Dodgson condensation. For any $n \times n$ matrix $A$, let $A_{r}(k, \ell)$ be the $r \times r$ connected submatrix whose upper leftmost corner is the entry $a_{k, \ell}$. If $\operatorname{det} A_{n-2}(2,2) \neq 0$ then

$$
\operatorname{det} A=\frac{\operatorname{det} A_{n-1}(1,1) \operatorname{det} A_{n-1}(2,2)-\operatorname{det} A_{n-1}(1,2) \operatorname{det} A_{n-1}(2,1)}{\operatorname{det} A_{n-2}(2,2)} .
$$

Applications of this method can be found in the papers of Amdeberhan and Zeilberger [1] and Zeilberger [29].

Proof of Theorem 4.1 for $m=1$. To prove

$$
\{n\}_{s, t}(q) \cdot\{n\}_{s, q t}(q)-\{n-1\}_{s, q t}(q) \cdot\{n+1\}_{s, t}(q)=(-t)^{n-1} q^{\left(\begin{array}{l}
n \\
2
\end{array}\right)},
$$

just apply equation (15) to the determinant for $\{n+1\}_{s, t}(q)$. The result is

$$
\{n+1\}_{s, t}(q)=\frac{\{n\}_{s, t}(q) \cdot\{n\}_{s, q t}(q)-(-1)^{n-1} t^{n-1} q^{\left(\begin{array}{c}
n \\
2
\end{array}\right)}}{\{n-1\}_{s, q t}(q)}
$$

and applying a little algebra finishes the proof.

It would be very interesting to prove the full version of Theorem 4.1 in a similar manner. This would perhaps require a more general version of condensation.

We now provide an application of the Euler-Cassini identity to infinite series. We will need the following slight variant of Theorem 4.1 when $q=1$. It can be proved by adapting Cigler's original proof.

Lemma 4.3. Let $r, m, n \in \mathbb{P}$ and $s, t$ be arbitrary integers. We have

$$
\{r n\} \cdot\{r(n+m-1)\}-\{r(n-1)\} \cdot\{r(n+m)\}=(-t)^{r(n-1)}\{r\}\{r m\} .
$$


Various authors have considered the following Fibonacci analogue of the Riemann zeta function

$$
\zeta_{F}(z)=\sum_{k=0}^{\infty} \frac{1}{F_{k}^{z}}
$$

See the article of Wu and Zhang [28] and references therein. In particular, there has been interest in finding estimates of the tails of such series for positive integers $z$. Holliday and Komatsu [17] considered what could be said for the Fibonacci polynomials where $t=1$ (recall (1)) and proved the following result.

Theorem 4.4 ([17]). If $t=1$ and $s, n \in \mathbb{P}$, then

$$
\left\lfloor\left(\sum_{k=n}^{\infty} \frac{1}{\{k\}_{s, 1}}\right)^{-1}\right\rfloor=\{n\}_{s, 1}-\{n-1\}_{s, 1}-\delta_{O}(n),
$$

and

$$
\left\lfloor\left(\sum_{k=n}^{\infty} \frac{1}{\{k\}_{s, 1}^{2}}\right)^{-1}\right\rfloor=s\{n\}_{s, 1}\{n-1\}_{s, 1}-\delta_{E}(n) .
$$

Holliday and Komatsu also asked if their theorem could be generalized to other $t$ and we will do this for the first summation. In addition, our results cover a more general class of sums and the proofs, based on Lemma 4.3, will be much simpler than the ones given in [17]. To see the equivalence of our second sum when $r=1$ with the one of Holliday and Komatsu, we note that if $t=1$ then

$$
\{n\}^{2}-\{n-1\}^{2}+(-1)^{n}=s\{n\}\{n-1\},
$$

an identity which is easily proved using Proposition 1.1 and equation (5).

Theorem 4.5. If $s \geq t \geq 1$ and $n, r \in \mathbb{P}$ then

$$
\left\lfloor\left(\sum_{k=n}^{\infty} \frac{1}{\{r k\}_{s, t}}\right)^{-1}\right\rfloor=\{r n\}_{s, t}-\{r(n-1)\}_{s, t}-\delta_{E}(r(n-1)) .
$$

If $t=1$ and $s, n, r \in \mathbb{P}$ then

$$
\left\lfloor\left(\sum_{k=n}^{\infty} \frac{1}{\{r k\}_{s, 1}^{2}}\right)^{-1}\right\rfloor=\{r n\}_{s, 1}^{2}-\{r(n-1)\}_{s, 1}^{2}-\delta_{E}(r(n-1)) .
$$

Proof. First note that both series must converge by comparison with the known convergent series $\sum_{k \geq 1} 1 / F_{k}$.

We now consider the first series. We will only give details for the case when $r(n-1)$ is even as the odd case is similar. For ease of notation, let

$$
A(n)=\sum_{k=n}^{\infty} \frac{1}{\{r k\}}
$$

It suffices to show that

$$
\{r n\}-\{r(n-1)\}-1 \leq \frac{1}{A(n)}<\{r n\}-\{r(n-1)\}
$$


Note that since $s, t$ are positive, so are all the $\{r k\}$ and inequalities will not be affected when multiplying by them.

We first deal with the right-hand inequality. Multiply through by $A(n)$ and then cancel the 1 on the right with the first term of the series $\{r n\} A(n)$. For $m \geq 1$ we then compare the term for $k=n+m$ in $\{r n\} A(n)$ with the term for $k=n+m-1$ in $\{r(n-1)\} A(n)$ to see that it suffices to show

$$
0<\frac{\{r n\}}{\{r(n+m)\}}-\frac{\{r(n-1)\}}{\{r(n+m-1)\}} .
$$

But this is true by Lemma 4.3 and the fact that $r(n-1)$ is even.

We now apply the same procedure as in the previous paragraph to the left-hand inequality and reduce it to proving

$$
\frac{\{r n\}}{\{r(n+m)\}}-\frac{\{r(n-1)\}}{\{r(n+m-1)\}} \leq \frac{1}{\{r(n+m-1)\}} .
$$

Cross-multiplying and using Lemma 4.3 again as well as the parity of $r(n-1)$, we see that it suffices to prove $t^{r(n-1)}\{r\}\{r m\} \leq\{r(n+m)\}$. Using Theorem 2.2 and the fact that $t$ is positive gives

$$
\{r(n+m)\} \geq\{r n+1\}\{r m\} \geq\{r(n-1)+2\}\{r\}\{r m\} \geq t^{r(n-1)+1}\{r\}\{r m\}
$$

where the last inequality comes from the fact that, by Proposition 2.1 and $s \geq t \geq 1$, we have $\{l\} \geq s^{l-1} \geq t^{l-1}$. Thus we are done with the first series.

The proof for the second series has many similarities, so we will only mention the places where they differ. Assume, again, that $r(n-1)$ is even. To obtain the squared version of (17), merely move the negative fraction onto the other side of the inequality (which we know to be true from the first half of the proof) and square both sides.

When each fraction in inequality (18) is replaced by its square one obtains, after clearing denominators,

$$
\{r n\}^{2}\{r(n+m-1)\}^{2}-\{r(n-1)\}^{2}\{r(n+m)\}^{2} \leq\{r n+r m\}^{2} .
$$

Factoring the left-hand side and applying Lemma 4.3 once again, this time with $t=1$, gives the equivalent inequality

$$
\{r\}\{r m\} \cdot(\{r n\}\{r(n+m-1)\}+\{r(n-1)\}\{r(n+m)\}) \leq\{r n+r m\}^{2} .
$$

It is easy to prove by induction that under the restrictions on $s, t$ the sequence $\{n\}$ is weakly increasing. Using this observation as well as repeated application of Theorem 2.2, we obtain

$$
\begin{aligned}
\{r(n+m)\}^{2} & =\{r(n+m)\} \cdot(\{r(n+m-1)\}\{r+1\}+\{r(n+m-1)-1\}\{r\}) \\
& \geq\{r n\}\{r m\}\{r(n+m-1)\}\{r\}+\{r(n+m)\}\{r(n-1)\}\{r m\}\{r\}
\end{aligned}
$$

and factoring out $\{r\}\{r m\}$ leads to the desired conclusion.

We believe that there are analogues of these results for other values of $s, t$. The restriction $(s, t) \neq(2,-1)$ in the following conjecture is to ensure that the series converges.

Conjecture 4.6. If $s>t \geq 1$ with $(s,-t) \neq(2,-1)$ and $n, r \in \mathbb{P}$ then

$$
\left\lfloor\left(\sum_{k=n}^{\infty} \frac{1}{\{r k\}_{s,-t}}\right)^{-1}\right\rfloor=\{r n\}_{s,-t}-\{r(n-1)\}_{s,-t}-1 .
$$

If $t=-1$ and $s, n, r \in \mathbb{P}$ then

$$
\left\lfloor\left(\sum_{k=n}^{\infty} \frac{1}{\{r k\}_{s,-1}^{2}}\right)^{-1}\right\rfloor=\{r n\}_{s,-1}^{2}-\{r(n-1)\}_{s,-1}^{2}-1 .
$$




\section{$5 \quad$ Fundamental properties of $\left\{\begin{array}{l}n \\ k\end{array}\right\}$}

We now return to the generalized Fibonomial coefficients. We will describe various recursions which they satisfy as well as analogues of the binomial theorem and Chu-Vandermonde summation.

It is not clear from the definition that $\left\{\begin{array}{l}n \\ k\end{array}\right\}$ is a polynomial in $s, t$ with nonnegative integral coefficients. This will follow by an easy induction using the first recursion in Theorem 5.2. The Lucanomials are not so well behaved. For instance,

$$
\left\langle\begin{array}{l}
4 \\
2
\end{array}\right\rangle=\frac{\left(s^{2}+3 t\right)\left(s^{4}+4 s^{2} t+2 t^{2}\right)}{s^{2}+2 t} .
$$

can not be brought to polynomial form. Also, we could introduce a $q$-analogue $\left\{\begin{array}{l}n \\ k\end{array}\right\}(q)$ of the generalized Fibonomials by using the the polynomials $\{n\}(q)$ defined by (14). But then it is easy to check that $\left\{\begin{array}{l}6 \\ 3\end{array}\right\}(q)$ is not a polynomial. This is one of the reasons we have decided to mainly consider the case $q=1$ in this work.

Our first property of generalized Fibonomials is their symmetry. This follows immediately from their definition.

Proposition 5.1. If $0 \leq k \leq n$ then

$$
\left\{\begin{array}{l}
n \\
k
\end{array}\right\}=\left\{\begin{array}{c}
n \\
n-k
\end{array}\right\}
$$

Next we consider two recursions for the Fibonomials.

Theorem 5.2. For $m, n \geq 1$ we have

$$
\begin{aligned}
\left\{\begin{array}{c}
m+n \\
m
\end{array}\right\} & =\{n+1\}\left\{\begin{array}{c}
n+m-1 \\
m-1
\end{array}\right\}+t\{m-1\}\left\{\begin{array}{c}
m+n-1 \\
m
\end{array}\right\} \\
& =Y^{n}\left\{\begin{array}{c}
n+m-1 \\
m-1
\end{array}\right\}+X^{m}\left\{\begin{array}{c}
m+n-1 \\
m
\end{array}\right\} .
\end{aligned}
$$

In particular they are polynomials in $s$ and $t$.

Proof. The first recursion follows easily from Theorem 2.2. The second can be obtained from the $q$-binomial recursion

$$
\left[\begin{array}{c}
m+n \\
m
\end{array}\right]=\left[\begin{array}{c}
m+n-1 \\
m-1
\end{array}\right]+q^{m}\left[\begin{array}{c}
m+n-1 \\
m
\end{array}\right]
$$

via the substitution (7).

The next result gives two analogues of the binomial theorem.

Theorem 5.3. Letting $z$ be an indeterminate, the genearlized Fibonomials satisfy

$$
\left(1+X^{n-1} z\right)\left(1+X^{n-2} Y z\right) \cdots\left(1+Y^{n-1} z\right)=\sum_{k=0}^{n}(-t)\left(\begin{array}{c}
k \\
2
\end{array}\right)\left\{\begin{array}{l}
n \\
k
\end{array}\right\} z^{k}
$$

and

$$
\frac{1}{\left(1-X^{n-1} z\right)\left(1-X^{n-2} Y z\right) \cdots\left(1-Y^{n-1} z\right)}=\sum_{k=0}^{\infty}\left\{\begin{array}{c}
n+k-1 \\
k
\end{array}\right\} z^{k} .
$$


Proof. We will indicate how to prove the first recursion as the second is similar. One approach is to use (20) and induction. Alternatively, one can start with the $q$-binomial theorem in the form

$$
(1+z)(1+q z) \cdots\left(1+q^{n-1} z\right)=\sum_{k=0}^{n} q^{\left(\begin{array}{c}
k \\
2
\end{array}\right)}\left[\begin{array}{l}
n \\
k
\end{array}\right] z^{k},
$$

substitute $Y^{n-1} z$ for $z$, use (7), and clear denominators. The details of both proofs are routine and so these are left to the reader.

It is interesting to note that when $t=-1$ we can write (22) using generalized Fibonomials with negative upper indices. We note that in this case, the generalized Fibonacci polynomial sequence is called an $\ell$-sequence (where $s=\ell$ ) and such sequences are intimately related with lecture hall partitions; see the papers by Bousquet-Mélou and Eriksson [2, 3] and by Savage and Yee [25] for more information. We first extend the sequence $\{n\}$ to negative integers by insisting that the recursion (2) continues to hold. In this case, it is not hard to show by induction that, for $n \geq 0$,

$$
\{-n\}=\frac{-\{n\}}{(-t)^{n}}
$$

So when $t=-1$ the $\{-n\}$ are polynomials in $s, t$ with integral coefficients. In this case, let

$$
\left\{\begin{array}{c}
-n \\
k
\end{array}\right\}_{s,-1}=\frac{\{-n\}\{-n-1\} \cdots\{-n-k+1\}}{\{k\} !}=(-1)^{k}\left\{\begin{array}{c}
n+k-1 \\
k
\end{array}\right\}_{s,-1} .
$$

Hence (22) becomes

$$
\frac{1}{\left(1-X^{n-1} z\right)\left(1-X^{n-2} Y z\right) \cdots\left(1-Y^{n-1} z\right)}=\sum_{k=0}^{\infty}\left\{\begin{array}{c}
-n \\
k
\end{array}\right\}_{s,-1}(-z)^{k} .
$$

The specialization $t=-1$ also permits us to obtain nice analogues of the formulas for the sum and alternating sum of a row of Pascal's triangle. This is because, by (5), we have $X Y=1$ which permits simplifications. Note also that if $s=2$ and $t=-1$ then it is easy to prove that $\langle n\rangle_{2,-1}=2$ for all $n \geq 0$, so that in this case the following identities reduce to the usual ones.

Corollary 5.4. If $t=-1$ then

$$
\sum_{k=0}^{n}\left\{\begin{array}{l}
n \\
k
\end{array}\right\}_{s,-1}=\left(1+\delta_{O}(n)\right) \prod_{i=1}^{\lfloor n / 2\rfloor}(2+\langle n-2 i+1\rangle)
$$

and

$$
\sum_{k=0}^{n}(-1)^{k}\left\{\begin{array}{l}
n \\
k
\end{array}\right\}_{s,-1}=\delta_{E}(n) \prod_{i=1}^{\lfloor n / 2\rfloor}(2-\langle n-2 i+1\rangle) .
$$

Proof. As usual, we just prove the first identity. Letting $t=-1$ and $z=1$ in equation (21) gives

$$
\sum_{k=0}^{n}\left\{\begin{array}{l}
n \\
k
\end{array}\right\}_{s,-1}=\left(1+X^{n-1}\right)\left(1+X^{n-2} Y\right) \cdots\left(1+Y^{n-1}\right)
$$

If $i<j$ then we have, using Proposition 1.1,

$$
\left(1+X^{i} Y^{j}\right)\left(1+X^{j} Y^{i}\right)=1+X^{i} Y^{i}\left(X^{j-i}+Y^{j-i}\right)+X^{i+j} Y^{i+j}=2+\langle j-i\rangle .
$$

Pairing up such factors and remembering that there will be an unpaired factor when $n$ is odd completes the proof. 
Returning to an arbitrary $t$, we can use any identity for $q$-integers and $q$-binomials to derive a corresponding one for $\{n\}$ and $\left\{\begin{array}{l}n \\ k\end{array}\right\}$. For example, the $q$-Chu-Vandermonde summation

$$
\left[\begin{array}{c}
m+n \\
k
\end{array}\right]=\sum_{i} q^{i(m-k+i)}\left[\begin{array}{c}
m \\
k-i
\end{array}\right]\left[\begin{array}{l}
n \\
i
\end{array}\right]
$$

gives rise to the following result.

Theorem 5.5. We have

$$
\left\{\begin{array}{c}
m+n \\
k
\end{array}\right\}=\sum_{i}(-t)^{i(i-k)} X^{m i} Y^{n(k-i)}\left\{\begin{array}{c}
m \\
k-i
\end{array}\right\}\left\{\begin{array}{c}
n \\
i
\end{array}\right\} .
$$

We end this section by mentioning that Proposition 2.4 can be used to prove various divisibility properties of the Fibonomials $\left\{\begin{array}{l}n \\ k\end{array}\right\}$. An example is the following primality testing condition. A proof for ordinary binomial coefficients can be found in the article of Dilcher and Stolarsky [11]. Because of Proposition 2.4, the demonstration carries over mutatis mutandis to the general case.

Proposition 5.6. Consider $s, t$ as variables. The positive integer $p$ is prime if and only if $\{p\}$ divides $\left\{\begin{array}{l}p \\ k\end{array}\right\}$ for each $0<k<p$.

\section{Catalan numbers}

In this section, we will consider an $s, t$-analogue of the Catalan numbers suggested by Louis Shapiro [private communication]. We will then investigate the 2-adic valuation of these generalized Catalan numbers for various values of $s$ and $t$, extending a well-known theorem in the case $s=$ $2, t=-1$.

Recall that the Catalan numbers are defined by

$$
C_{n}=\frac{1}{n+1}\left(\begin{array}{c}
2 n \\
n
\end{array}\right)
$$

for $n \geq 0$. By analogy, define the generalized Catalan numbers to be

$$
C_{\{n\}}=\frac{1}{\{n+1\}}\left\{\begin{array}{c}
2 n \\
n
\end{array}\right\} .
$$

Ekhad [13] was the first to note that these must be polynomials in $s, t$ with nonnegative integral coefficients, because setting $m=n$ in Theorem 2.2 and doing some algebraic manipulation shows that

$$
C_{\{n\}}=\left\{\begin{array}{c}
2 n-1 \\
n-1
\end{array}\right\}+t\left\{\begin{array}{c}
2 n-1 \\
n-2
\end{array}\right\} .
$$

So we can ask about the arithmetic properties of $C_{\{n\}}$ for integers $s, t$.

To state and prove our results, we will need some notation. First of all, let

$$
\zeta_{\mathbf{b}}(n)=\text { the number of nonzero digits of } n \text { in the base } \mathbf{b} \text {. }
$$

Also, let

$$
\kappa_{\mathbf{b}}(m+n)=\text { the number of carries in doing the addition }(m)_{\mathbf{b}}+(n)_{\mathbf{b}} \text {. }
$$

It will also be useful to introduce the expansion of $n$ in an unusual base for the integers. Given any infinite increasing sequence of positive integers $\mathbf{b}=\left(b_{0}, b_{1}, \ldots\right)$ such that $b_{0}=1$ and $b_{i} \mid b_{i+1}$ for $i \geq 1$ we consider the expansion of $n$ in base $\mathbf{b}$ to be $n=\sum_{i \geq 0} n_{i} b_{i}$ where $0 \leq n_{i}<b_{i+1} / b_{i}$ for 
all $i \geq 0$. We will utilize the shorthand $(n)_{\mathbf{b}}=\left(n_{0}, n_{1}, \ldots\right)$ for the digits $n_{i}$ in this expansion. If b consists of powers of an integer $m>1$ then we will merely write $(n)_{m}$. And we will omit the subscript entirely if the base is clear from context. A particular base of interest to us is the one where

$$
\mathbf{F}=\left(1,3,3 \cdot 2,3 \cdot 2^{2}, 3 \cdot 2^{3}, \ldots\right) .
$$

This base arises naturally from studying the fractal nature of the Fibonomial triangle modulo 2. See the paper of Chen and Sagan [5] for details. Other number-theoretic functions which depend on choosing a base will follow the same conventions.

The next result is well-known. See the paper by Deutsch and Sagan [10] for a (mostly) combinatorial proof.

Theorem 6.1. If $C_{n}$ is an ordinary Catalan number then

$$
\nu_{2}\left(C_{n}\right)=\zeta_{2}(n+1)-1
$$

We now prove analogues of this theorem for $C_{\{n\}}$ using results from Section 3, We first recall a famous theorem of Kummer [19] which is needed in the sequel.

Theorem 6.2. For any prime, $p$, we have

$$
\nu_{p}\left(\left(\begin{array}{c}
m+n \\
n
\end{array}\right)\right)=\kappa_{p}(m+n) .
$$

We start by considering what happens if $s$ and $t$ are both odd.

Theorem 6.3. Let $s$ and $t$ be odd. We have

$$
\nu_{2}\left(C_{\{n\}}\right)= \begin{cases}\zeta_{\mathbf{F}}(n+1)+\nu_{2}(\{6\})-3 & \text { if } t \equiv 1(\bmod 4) \text { and } n \equiv 3,4(\bmod 6), \\ \zeta_{\mathbf{F}}(n+1)-1 & \text { else. }\end{cases}
$$

Proof. Since

$$
\nu_{2}\left(C_{\{n\}}\right)=\nu_{2}(\{2 n\} !)-\nu_{2}(\{n\} !)-\nu_{2}(\{n+1\} !),
$$

we can apply Corollary 3.2 . As usual, we will just supply details when $t \equiv 3(\bmod 4)$. In this case, the terms from the corollary containing a factor of $\nu_{2}(\{3\})$ cancel in the above equation. As a result,

$$
\nu_{2}\left(C_{\{n\}}\right)=\nu_{2}\left(\left(\begin{array}{c}
\lfloor 2 n / 3\rfloor \\
\lfloor n / 3\rfloor
\end{array}\right)\right)= \begin{cases}\zeta_{2}(\lfloor n / 3\rfloor) & \text { if } n \equiv 0,1(\bmod 3) \\
\zeta_{2}(\lfloor n / 3\rfloor+1)-1 & \text { if } n \equiv 2(\bmod 3)\end{cases}
$$

where the second equality comes from Kummer's Theorem and the fact that when adding $k$ to itself in base two the number of carries is the number of nonzero digits. We now translate from base 2 to base $\mathbf{F}$. We must consider the congruence classes modulo three individually. We will do $n \equiv 0(\bmod 3)$ and leave the rest to the reader. In this case $n=3 k$ where $(k)_{2}=\left(k_{0}, k_{1}, \ldots\right)$. So $(n+1)_{\mathbf{F}}=(3 k+1)_{\mathbf{F}}=\left(1, k_{0}, k_{1}, \ldots\right)$. Thus

$$
\zeta_{2}(\lfloor n / 3\rfloor)=\zeta_{2}(k)=\zeta_{\mathbf{F}}(3 k+1)-1=\zeta_{\mathbf{F}}(n+1)-1
$$

which is what we wished to prove.

Now we consider $s$ and $t$ of opposite parity. As remarked before, if $s$ is odd and $t$ is even then $C_{\{n\}}$ is always odd. If $s$ is even and $t$ is odd then Corollary 3.4 and Theorem 6.1 also make evaluation a simple matter. Thus we arrive at the following theorem. 
Theorem 6.4. Let $s$ and $t$ be of opposite parity. We have

$$
\nu_{2}\left(C_{\{n\}}\right)= \begin{cases}\zeta_{2}(n+1)-1 & \text { if } t \text { is odd, } \\ 0 & \text { ift is even. }\end{cases}
$$

We conclude with a very interesting question that Shapiro raised when he defined the Catalans $C_{\{n\}}$. As mentioned earlier, there is a nice combinatorial interpretation of $\left\{\begin{array}{l}n \\ k\end{array}\right\}$ using tilings [24]. But it remains an open problem to find a combinatorial interpretation for $C_{\{n\}}$. This is especially puzzling given the plethora of combinatorial interpretations for the ordinary Catalan numbers.

\section{References}

[1] Tewodros Amdeberhan and Doron Zeilberger. Determinants through the looking glass. Adv. in Appl. Math., 27(2-3):225-230, 2001. Special issue in honor of Dominique Foata's 65th birthday (Philadelphia, PA, 2000).

[2] Mireille Bousquet-Mélou and Kimmo Eriksson. Lecture hall partitions. Ramanujan J., 1(1):101-111, 1997.

[3] Mireille Bousquet-Mélou and Kimmo Eriksson. Lecture hall partitions II. Ramanujan J., 1(2):165-185, 1997.

[4] Francesco Brenti. Log-concave and unimodal sequences in algebra, combinatorics, and geometry: an update. In Jerusalem combinatorics '93, volume 178 of Contemp. Math., pages 71-89. Amer. Math. Soc., Providence, RI, 1994.

[5] Xi Chen and Bruce E. Sagan. On the fractal nature of Fibonomial coefficients. In preparation.

[6] Johann Cigler. A new class of q-Fibonacci polynomials. Electron. J. Combin., 10:Research Paper 19, 15 pp., 2003.

[7] Johann Cigler. q-Fibonacci polynomials. Fibonacci Quart., 41(1):31-40, 2003.

[8] Johann Cigler. Some algebraic aspects of Morse code sequences. Discrete Math. Theor. Comput. Sci., 6(1):55-68 (electronic), 2003.

[9] Johann Cigler. q-Fibonacci polynomials and the Rogers-Ramanujan identities. Ann. Comb., 8(3):269-285, 2004.

[10] Emeric Deutsch and Bruce E. Sagan. Congruences for Catalan and Motzkin numbers and related sequences. J. Number Theory, 117(1):191-215, 2006.

[11] Karl Dilcher and Kenneth B. Stolarsky. A Pascal-type triangle characterizing twin primes. Amer. Math. Monthly, 112(8):673-681, 2005.

[12] C. L. Dodgson. Condensation of determinants, being a new and brief method for computing their arithmetic values. Proc. Royal Soc. of London, 15:150-155, 1866.

[13] Shalosh Ekhad. The Sagan-Savage Lucas-Catalan polynomials have positive coefficients. Preprint http://www.math.rutgers.edu/ zeilberg/mamarim/mamarimhtml/bruce.html.

[14] Adam M. Goyt and David Mathisen. Permutation statistics and q-Fibonacci numbers. Electron. J. Combin., 16(1):Research Paper 101, 15 pp., 2009. 
[15] Adam M. Goyt and Bruce E. Sagan. Set partition statistics and q-Fibonacci numbers. European J. Combin., 30(1):230-245, 2009.

[16] Verner E. Hoggatt, Jr. and Calvin T. Long. Divisibility properties of generalized Fibonacci polynomials. Fibonacci Quart., 12:113-120, 1974.

[17] Sarah H. Holliday and Takao Komatsu. On the sum of reciprocal generalized Fibonacci numbers. Integers, 11(4):441-455, 2011.

[18] Thomas Koshy. Fibonacci and Lucas numbers with applications. Pure and Applied Mathematics (New York). Wiley-Interscience, New York, 2001.

[19] E. E. Kummer. Über die Ergänzungssätze zu den allgemeinen reciprocitätsgesetzen. J. reine angew. Math., 44:93-146, 1852.

[20] Edouard Lucas. Theorie des Fonctions Numeriques Simplement Periodiques. Amer. J. Math., 1(2):184-196, 1878.

[21] Edouard Lucas. Theorie des Fonctions Numeriques Simplement Periodiques. [Continued]. Amer. J. Math., 1(3):197-240, 1878.

[22] Edouard Lucas. Theorie des Fonctions Numeriques Simplement Periodiques. Amer. J. Math., 1(4):289-321, 1878.

[23] Victor H. Moll. Numbers and functions. Student Mathematical Library. American Mathematical Society, Providence, RI, 2012. Special Functions for Undergraduates.

[24] Bruce E. Sagan and Carla D. Savage. Combinatorial interpretations of binomial coefficient analogues related to Lucas sequences. Integers, 10:A52, 697-703, 2010.

[25] Carla D. Savage and Ae Ja Yee. Euler's partition theorem and the combinatorics of $\ell$ sequences. J. Combin. Theory Ser. A, 115(6):967-996, 2008.

[26] Richard P. Stanley. Log-concave and unimodal sequences in algebra, combinatorics, and geometry. In Graph theory and its applications: East and West (Jinan, 1986), volume 576 of Ann. New York Acad. Sci., pages 500-535. New York Acad. Sci., New York, 1989.

[27] Herbert S. Wilf. generatingfunctionology. Academic Press Inc., Boston, MA, second edition, 1994.

[28] Zhengang Wu and Wenpeng Zhang. Several identities involving the Fibonacci polynomials and Lucas polynomials. J. Inequal. Appl., 205, 14 pp., 2013.

[29] Doron Zeilberger. Reverend Charles to the aid of Major Percy and Fields medalist Enrico. Amer. Math. Monthly, 103(6):501-502, 1996. 